\title{
EchoGéo
}

$37 \mid 2016$

Femmes et migrations : celles qui restent

\section{Dialogue sur la condition féminine : regards croisés de géographes}

Entretien entre Elizabeth Crémieu et Emilie Ponceaud-Goreau

Élizabeth Crémieu and Émilie Ponceaud-Goreau

\section{OpenEdition}

\section{Journals}

Electronic version

URL: https://journals.openedition.org/echogeo/14672

DOI: $10.4000 /$ echogeo. 14672

ISSN: 1963-1197

Publisher

Pôle de recherche pour l'organisation et la diffusion de l'information géographique (CNRS UMR 8586)

Electronic reference

Élizabeth Crémieu and Émilie Ponceaud-Goreau, "Dialogue sur la condition féminine : regards croisés de géographes", EchoGéo [Online], 37 | 2016, Online since 07 October 2016, connection on 10 August 2021. URL: http://journals.openedition.org/echogeo/14672 ; DOI: https://doi.org/10.4000/echogeo. 14672

This text was automatically generated on 10 August 2021

EchoGéo est mis à disposition selon les termes de la licence Creative Commons Attribution - Pas d'Utilisation Commerciale - Pas de Modification 4.0 International (CC BY-NC-ND) 


\title{
Dialogue sur la condition féminine : regards croisés de géographes
}

\author{
Entretien entre Elizabeth Crémieu et Emilie Ponceaud-Goreau \\ Élizabeth Crémieu and Émilie Ponceaud-Goreau
}

1 Elizabeth Crémieu, est agrégée de géographie, professeur en classes préparatoires de 1986 à 2011 et maître de conférences à SciencesPo Paris de 1989 à 2009. Elle a publié Géopolitique de la condition féminine dans le monde en 2014, ouvrage dont il est question dans l'entretien qui suit. Émilie Ponceaud-Goreau est doctorante en géographie humaine à l'Université de Bordeaux-Montaigne et ses travaux portent sur l'émergence et la diffusion de la préscolarisation au Tamil Nadu et à Pondichéry.

2 - Élizabeth Crémieu (EC). Géopolitique de la Condition féminine est une tentative de synthèse sur la condition féminine dans le monde actuel. Il se décompose en trois parties: l'infériorisation des femmes, héritage universel; les luttes et les progrès, incontestables, remarquables; enfin les problèmes et les régressions. Il s'articule autour de trois idées : la lutte entre tradition et modernité est plus tendue que jamais ; partout les acquis des femmes sont insuffisants et fragiles; et partout la condition féminine est une question politique et géopolitique. Quant au site Internet http:// abc.immigration.free.fr, c'est une initiative plus ancienne. C'est un site qui présente des témoignages de personnes ayant immigré en France à l'âge adulte, à partir de témoignages recueillis entre 2001 et 2008. L'idée était de lutter contre les stéréotypes opposés du migrant-victime et du migrant-profiteur, et j'ai compris au fil des interviews que les migrants étaient des combattants. La moitié des personnes interviewées sont des femmes.

3 - Emilie Ponceaud-Goreau (EPB). Sur la quatrième de couverture, on peut lire que « cet ouvrage s'adresse à ceux qui veulent connaître les origines des inégalités entre les hommes et les femmes et étudier les enjeux de pouvoir qui en découlent à l'ère de la mondialisation ». L'information est dense, bien structurée, plutôt à visée pédagogique mais le livre s'adresse aussi aux amateurs de géographie radicale en s'interrogeant sur les rôles de domination. Comment le présenteriez-vous? 
4 - EC. Ce livre présente la situation des femmes comme un combat entre la tradition, qui reste dominante dans un pays comme l'Inde, et la modernité. Nous vivons en ce moment une lutte des femmes contre les traditions qui les infériorisent, en faveur de leur autonomie, en sachant que toutes les femmes ne sont pas dans ce combat. Elles ne sont pas toutes féministes et certaines s'accommodent extrêmement bien de leur statut. Et n'oublions pas que les premiers féministes étaient des hommes, en Inde on peut citer Rammohun Roy et Rabindranath Tagore La modernité s'accompagne de l'idée que les femmes sont les égales des hommes et qu'elles ont droit à l'autonomie ; c'est une lutte qui s'effectue à tous les niveaux : l'individu, la famille, la société, au niveau de chaque pays, dans le politique, dans le religieux - et c'est une lutte qui entraîne de la part des tenants du patriarcat des violences. Aujourd'hui, vous avez des réactions violentes des traditionalistes contre la volonté d'autonomisation des femmes, dans les campagnes du Tiers monde mais parfois aussi dans nos pays.

5 Le sujet des femmes est un sujet politique. Ainsi en ce qui concerne le microcrédit, je parle de la position des économistes et des géographes radicaux qui sont contre le microcrédit en expliquant qu'il va de pair avec le libéralisme et la réduction des subventions de l'État, que cela revient à dire aux pauvres que «si vous n'y arrivez pas, c'est de votre faute, tant pis pour vous "... Mais selon vous, Emilie, le micro-crédit est-il développé au Tamil Nadu et si oui, a-t-il un impact notable?

6 - EPG. Isabelle Guérin (2015), qui a longuement étudié la question de la micro-finance au Tamil Nadu, souligne que la micro-finance actuelle a tendance à reproduire les logiques de domination et d'inégalités car le ciblage vise l'efficacité des remboursements plus que le bouleversement politique des rapports de genre et les bénéficiaires restent une minorité relativement privilégiée. De mon côté, j’ai rencontré à Chennai et à Pondichéry des associations menées par des acteurs civils et des ONG qui ont un rôle indéniable dans la réalisation des petits projets qui s'adressent plus directement aux femmes même si l'on peut toujours s'interroger sur le ciblage des bénéficiaires. Celles de Chennai bénéficient de l'argent des philanthropes locaux et s'orientent vers les nombreuses familles migrantes venus des États voisins ou des campagnes. À Pondichéry, les catholiques et les associations fondées par les étrangers essaient aussi de contrebalancer le poids du chef de famille, qui reste le principal récepteur des aides du gouvernement. Cela a été particulièrement criant lors de la distribution des aides financières et des logements aux personnes touchées par le tsunami de 2004. L'aide internationale a été très importante. Le gouvernement a choisi de maîtriser la redistribution et les critères retenus, notamment la signature du chef de famille, ont écarté certaines bénéficiaires, comme les femmes et les mères de famille séparées de leur conjoint. Les associations présentes localement ont alors cherché des micro-solutions: logement dans une partie de l'école gérée par l'ONG, achat de machines à coudre ou mise à disposition d'une salle pour que les femmes puissent effectuer des massages...

7 Au cours de la lecture de votre livre, on est saisi par la persistance du sentiment d'infériorité de la femme, on s'interroge autant sur le sexisme ordinaire dans les pays des Nords que sur les décalages fondamentaux qui peuvent exister entre les femmes des pays des Nords et celles des pays des Suds. Comment situez-vous le curseur entre tolérance face à une culture différente et indignation face à des choses qui, où qu'elles se passent, restent inadmissibles? 
8 - EC. Toute tradition doit être réexaminée à la lumière de l'analyse critique... Je pense qu'il faut s'indigner. Il y a des féministes dans les pays du Sud qui s'indignent, qui publient des ouvrages, des blogs et qui créent des associations, des refuges, et ces femmes-là se mettent en danger. Il faut s'indigner avec elles, je suis contre le relativisme culturel, je déteste que l'on me dise que toutes les cultures se valent. Je juge les cultures à la place qu'elles donnent aux femmes, mon curseur à moi est vers l'indignation. Il faut penser à toutes ces femmes qui se mettent en danger, il y a de véritables héroïnes, par exemple Nawal El Sadaoui. Elle doit avoir quatre-vingts ans : médecin, elle a été licenciée de l'hôpital où elle travaillait en Égypte car elle luttait contre l'excision; elle s'est élevée contre les islamistes, les deux combats étaient liés, elle a été condamnée pour apostasie et divorcée de force. J'admire beaucoup Taslima Nasreen, la femme médecin bangladeshi qui vit cachée en exil, ou Norma Cruz, l'activiste guatémaltèque, qui reçoit des menaces parce qu'elle protège des femmes battues, ou Lydia Cacho, la Mexicaine. Et je pourrais en citer beaucoup d'autres. Toutes ces femmes luttent contre la culture machiste et nous devons nous indigner et lutter à leurs côtés.

9 - EPG. «Vivre selon la tradition » est le nom d'une de vos parties. Je pense aux femmes indiennes marquées par les figures féminines du panthéon hindou : la fidélité de Sita, la générosité de Lakshmi, la qualité maternelle de Shakti, l'intelligence de Parvati mais aussi la combativité et la violence de Durga et de Kali. Dans les années 1920, Gandhi utilise la symbolique féminine pour mobiliser les Indiennes. Il leur demande de faire vœu de "swadeshi », c'est-à-dire de renoncer aux biens étrangers et chaque jour, se consacrer à filer le coton. Il les encourage à tenir des piquets de grève devant les magasins vendant des produits importés car elles ont « la patience » de le faire. Il leur donne « un rôle nouveau, mais fondé sur une conception traditionnelle idéalisée de la femme » (Tawa-Lama Rewal, 2004, p. 37). C'est sur elles encore que repose le « fardeau » du prestige de la caste, une responsabilité qui leur donne un réel pouvoir au sein de la sphère domestique (notamment à travers la préparation des repas et les rituels religieux) mais les oblige dans le même temps à préserver la pureté de la famille. Cette représentation idéalisée des rôles familiaux dévolus aux femmes provoque un contrôle social très fort. Elles connaissent depuis l'enfance les contraintes d'être née femme, les dominations, les incertitudes, l'insécurité ou les restrictions à la mobilité. La famille est souvent leur plus grand danger : violence domestique, fœeticide, sous-alimentation et manque de soins, viols, crimes pour dot. Et pourtant, lors des entretiens menés pendant mes recherches doctorales, cette vie dure est rarement présentée comme misérable, elles sont dignes et courageuses et elles ont foi dans le progrès matériel et social. Comment voyez-vous les choses?

- EC. Cela me fait penser à Viramma, l'héroïne du livre de Josiane Racine (1994) Une vie paria : le rire des asservis, Inde du Sud. Ce livre est extraordinaire, j'ai calculé que Viramma était née en 1938. Chaque fois que j'ai rencontré des femmes qui avaient une vie très dure, elles ne se plaignaient pas, elles étaient plutôt dans la satisfaction d'avoir affronté et surmonté les difficultés. Ces femmes croient en l'éducation pour libérer leurs enfants et spécialement leurs filles : leur bonheur tient à la projection qu'elles font sur leurs enfants d'une vie meilleure, c'est une satisfaction de leur existence. Je repense à ce que me disait Camla (interviewée dans le cadre du projet abc.immigration.free.fr) : «Je dis aux enfants : je vais me battre, je vais travailler nuit et jour pour que vous ayez un diplôme, pour que vous soyez pas obligés de faire ce que je fais ». C'était une femme heureuse, elle vivait son statut difficile sans la moindre 
plainte, elle était dans la réalisation de son projet de vie. Je suis frappée par la dignité des personnes qui ont des vies extrêmement difficiles, le fait qu'elles ne se plaignent pas, au contraire elles savent apprécier le peu qu'elles ont et le peu qu'elles gagnent, s'amusent et plaisantent et se saisissent de toutes les occasions de rire. C'est une réaction vitale, elles veulent aimer la vie, elles pratiquent l'humour et se défendent par l'humour. Il y a cette force vitale, cette dignité, ce courage chez les personnes que j'ai interviewées. Qui trouvent souvent un secours dans la religion. Comme j'établis toujours des comparaisons entre la France et les autres pays, je pense aux livres de Marie Rouanet, par exemple La marche lente des glaciers : dans sa famille extrêmement pauvre, la religion donnait de la beauté et de la dignité à l'existence, ses parents étaient catholiques comme on respire et c'était une source d'équilibre, de sérénité et même de dignité, la beauté dans leur vie était liée en grande partie à la religion, c'était une sorte d'équilibre, d'harmonie des pratiques religieuses. La religion apporte un soutien à des gens très modestes et à des femmes défavorisées. D'ailleurs, de nombreux spécialistes expliquent le regain de religiosité en Afrique par l'aggravation de la situation économique des plus pauvres : citons Tanella Boni dans Que vivent les femmes d'Afrique? paru en 2008, ou Abel Kouvouama, dans Modernité africaine. Figures du politique et du religieux paru en 2002.

Quant à vous, au fil de vos séjours sur le terrain, avez-vous constaté une amélioration de la situation des femmes? Car les statistiques indiennes sont inquiétantes : foeticides dans le Nord-ouest, surmortalité féminine, en particulier mortalité maternelle, mariages précoces, violences, etc. L'Inde est très diverse, quelle est la situation au Tamil Nadu?

11 - EPG. La situation des femmes est nettement plus favorable dans les États du Tamil $\mathrm{Nadu}$ et du Kerala, toute une série d'indicateurs généraux le confirme et personnellement je le constate auprès des familles de jeunes enfants. Si la baisse de la fécondité est bien moins rapide en Inde que dans d'autres pays d'Asie, à commencer par la Chine, la mortalité infantile n'est plus que de 1,2\% au Kerala à 5,6\% au Madhya Pradesh en 2012. Le suivi de la grossesse et de la prime enfance y est assuré gratuitement par le réseau des centres médicaux publics. L'État du Tamil Nadu a investi également dans l'éducation des jeunes filles et donc des futures mères. La corrélation négative entre fécondité et alphabétisation est vérifié dans cet État: le nombre d'enfants par femme ne serait que de 1,7. Le comportement des classes moyennes sera aussi réellement décisif car les parents des familles les plus modestes aspirent à y appartenir et cherchent à en acquérir les marqueurs. Les familles investissent dans l'éducation de tous leurs enfants, notamment en choisissant des écoles privées. Bien sûr, certaines sont non-mixtes, le biais genre peut-être décisif dans le choix de l'école mais pas davantage que le rang dans la fratrie. Il faut aussi prendre en compte l'importance dans l'éducation des institutions catholiques, des associations et des ONG qui ont une réputation de qualité et qui enseignent souvent en anglais dès le plus jeune âge. Elles sont souvent plutôt disposées à accueillir les fillettes et communiquent autour de cela avec leurs donateurs qu'ils soient philanthropes indiens ou étrangers. Il faut penser également que la population est plutôt jeune donc peut-être plus encline à adopter des attitudes positives en faveur de l'émancipation des femmes. Des mesures politiques sont aussi prises pour que la voix féminine soit mieux entendue comme la réservation d'un tiers des sièges aux femmes dans les comités de panchayats ${ }^{1}$ et l'équivalent des conseils d'école. 
12 - EC. Dans ce pays fédéral, comment se fait le partage des politiques de genre entre État central et État local?

13 - EPG. Les politiques de genre se mêlent aux mesures socio-économiques diverses et beaucoup sont officiellement des compétences partagées. Il y a cependant une grande différence entre les idées impulsées par New Delhi et la manière dont elles sont mises en place dans les États. Le Tamil Nadu a des préoccupations sociales avancées et un souci de visibilité de sa politique. Par exemple dans le domaine de l'éducation : dans les années 1990, des incitations financières étaient versées aux directeurs d'écoles qui avaient les plus forts taux de scolarisation féminine, les jeunes filles qui entraient dans le secondaire recevaient l'emblématique vélo rose, parfois mauve, associé à la non moins emblématique ministre en chef du Tamil Nadu Jayalalitha. En août 2015, l'assistant elementary education à Marakkanam (district de Villupuram, Tamil Nadu) m'indiquait lors d'un entretien que la part des femmes enseignantes dans les écoles primaires de ce district, qui compte 380 enseignants, était devenue majoritaire par rapport aux hommes car, depuis plusieurs années, seules des femmes y avaient été nouvellement recrutées. Les recruteurs mettent en avant leurs qualités « maternelles». Elles sont surtout aux yeux des mères de familles des interlocutrices plus accessibles que leurs homologues masculins. Je crois que leur statut et leur réussite professionnelle encouragent également la poursuite des études des filles.

14 - EC. La condition féminine au Tamil Nadu, sur votre terrain, s'améliore-t-elle? Sur quels points et pour quelles raisons ? L'action des féministes y est-elle importante ?

15 - EPG. Le Tamil Nadu est un État précurseur dans bien des domaines, par exemple,combattre la faim des enfants et encourager la scolarisation sont devenues des priorités au Tamil Nadu bien avant qu'une loi du gouvernement central n'intervienne. Dès les années 1950, de nombreuses écoles et centres de soins fournissaient des repas chauds aux enfants. Mes recherches concernent plus particulièrement la diffusion de la préscolarisation des enfants de moins de six ans : imaginez que plus de 54000 centres publics fonctionnent dans tout l'État, offrant un mode de garde gratuit et sécurisé, des bases d'enseignement et ce fameux repas du midi aux enfants qui ne sont pas encore scolarisés. Certains adversaires politiques accusent le gouvernement de soutenir des mesures populistes mais concrètement, cela ouvre des perspectives nouvelles pour des mères de familles extrêmement courageuses. Elles peuvent travailler plus librement, ce qui augmente le revenu du foyer et modifie le rapport de forces intrafamilial. Les filles aînées peuvent poursuivre leur scolarité sans devoir s'arrêter pour garder les cadets. La préscolarisation réduit les inégalités de genre sur la longueur du parcours scolaire des fillettes. Elles sont en meilleure santé et intégrées très tôt dans le cursus. Les établissements préscolaires, publics ou privés - car l'attractivité du privé est importante dans toutes les couches de la société - sont aussi des espaces ouverts à la voix féminine. Les mères s'y rencontrent ou y trouvent des conseils. Les nombreuses enseignantes ont joué pour cela un rôle important : elles sont de véritables médiatrices entre les familles et les institutions, les mères de familles se sentent plus libres de parler avec elles qu'avec les enseignants du primaire. Leur statut et leur réussite professionnelle encouragent également la poursuite des études des filles, renforçant cette boucle de rétroaction positive. L'éducation reste une priorité pour toutes les familles. Les mères que j'interviewe sont incroyables et très dévouées: elles économisent pour offrir des cours du soir à leurs enfants et mettent tout en œuvre pour leur ouvrir les portes des meilleures écoles possibles. Leurs journées sont 
tellement longues: elles se lèvent avant tout le monde pour aller acheter le lait qui servira au chaï (thé au lait et aux épices), chercher l'eau à la pompe publique, nettoyer la maison, préparer le repas avant d'amener les enfants à l'école et d'aller travailler comme domestique... Les mères les plus modestes ont vraiment foi en l'éducation de leurs enfants. Les investissements publics dans le secondaire et le supérieur contribuent certainement à cette réussite. Cela ouvre de nouveaux défis.

16 - EC. Comment au niveau de l'Inde aborde-t-on la question féminine et cherche-t-on à améliorer le rôle des femmes?

17 - EPG. On parle souvent de l'Inde par son actualité négative. Mais, il faut aussi mettre en avant les points positifs. Très vite, les femmes indiennes manifestent et revendiquent leur liberté de s'instruire, de tomber amoureuse, de mener leur vie sans être jugées, de vivre sans la peur d'être agressée à l'opposé d'une actualité tragique comme cette étudiante victime d'un viol collectif dans un bus à New Delhi le 19 décembre 2012. C'est difficile de définir exactement le statut de la femme indienne au vu des multiples formes du patriarcat indien. Nous pouvons choisir une approche par le droit et souligner que le premier mouvement indien des femmes naît du mouvement nationaliste dès la première moitié du $\mathrm{XX}^{\mathrm{e}}$ siècle et que la Constitution dès 1950 inscrit l'égalité devant la loi, la liberté de pensée, d'expression et de la religion, la prohibition de «toute discrimination fondée sur la religion, la race, la caste, le sexe ou le lieu de naissance ». La deuxième vague du mouvement indien des femmes dans les années 1970 les place en tant qu'actrices incontournables des enjeux du développement. Sur le plan politique, nous pouvons aussi rappeler qu'Indira Gandhi est premier ministre en 1966 ou que des quotas permettent de lutter contre l'invisibilité politique (la décentralisation introduit un système de quotas de $33 \%$ des sièges réservés aux femmes mais chaque situation est discutée).

18 Et vous, pensez-vous que les quotas peuvent représenter un effet d'opportunité suffisant? Et quand Stéphanie Tawa-Lama Rewal (2004) s'interroge sur les femmes qui souvent, en Inde, au Sri Lanka, au Pakistan, au Bengladesh accèdent au pouvoir en qualité d'héritières, pensez-vous que l'on puisse dire qu'elles sont l'arbre qui cache la forêt?

19 - EC. Les femmes qui accèdent au pouvoir en qualité d'héritières sont plutôt l'arbre qui cache l'absence de forêt! Mais la situation évolue partout: il y a de plus en plus de femmes élues, certaines occupant des postes politiques importants et ceci dans beaucoup de pays. Les quotas y sont pour quelque chose, et même si le système des quotas peut être critiqué comme toute mesure de discrimination positive (je préfère l'expression américaine : affirmative action), moi qui suis pragmatique, j'y suis favorable.

20 - EPG. «Le féminisme indien a deux siècles " titre Martine Van Woerkens (2010). Des universitaires et des écrivaines animent le débat et des militantes investissent le terrain politique tandis que d'autres sont en contact direct avec les femmes. Pensezvous que le féminisme dans les pays des Suds puisse être une " révolution pacifique »?

21 - EC. Le féminisme dans les pays du Sud est une révolution: elle est pacifique du côté des femmes, Benoîte Groult dit que « le féminisme n’a jamais tué personne alors que le machisme, lui, tue tous les jours ". Être féministe dans certains pays du Sud est très risqué, d'ailleurs dans le livre j'ai cité l'histoire d'une femme en Inde il y a 20 ans qui avait cherché à empêcher des mariages d'enfant et que le panchayat avait condamnée à subir un viol collectif. Il y a quelques mois seulement, un garçon était accusé d'avoir été en relation avec une femme de plus haute caste et ses sœurs ont été condamnées à 
subir un viol collectif. Et ça s'est passé cette année. J'ai beaucoup lu sur le viol. Le viol n'est pas toujours le résultat d'une pulsion sexuelle irrépressible d'un homme, souvent c'est plutôt une façon qu'a la société de punir une femme d'avoir été déviante, c'est souvent une punition infligée par la société à la femme qui est sortie de son rôle traditionnel. La jeune fille violée en décembre 2012 sortait du cinéma avec son copain et pour ces brutes qui ont volé le bus et l'ont violée, c'était une transgression. Ratna Kapur, professeure de droit à Delhi, à Yale et Cambridge, décrit avec lucidité et intelligence la violence des hommes de basse condition à l'égard des femmes plus favorisées : la seule supériorité qui leur restait, celle d'être des hommes, leur a été enlevée. Plus ces hommes sont socialement en échec, plus ils sont agressifs à l'égard des femmes plus favorisées et autonomes. Ça les rend enragés. C'est vrai dans beaucoup de pays.

22 Ce qui m'amène au viol conjugal: dans les sociétés traditionnelles, c'est le mari qui décide et la femme n'a pas le droit de refuser les relations conjugales. Pratiquement toutes les sociétés traditionnelles fonctionnent comme cela. Chez nous aussi, jusqu'en 1980, si la femme disait non c'était une violation de contrat et elle se mettait en faute. On a l'impression qu'il y a un abîme entre les femmes en Inde et les femmes chez nous. Pourtant, il ne faut pas oublier qu'il y a un siècle, les femmes étaient placées sous l'autorité de leur mari (selon le Code Napoléon) et les enfants sous l'autorité paternelle, qu'une femme ne pouvait travailler sans autorisation de son mari, que si elle épousait un étranger elle perdait sa nationalité ce qui n'était pas le cas d'un homme épousant une étrangère, etc. Il faut imaginer l'énorme chemin parcouru en moins de cent ans : nous approchons de la fin du chemin et les femmes indiennes sont au début. Mais nulle part les femmes ne sont arrivées où l'on doit arriver.

23 - EPG. L'expérience que j'ai acquise sur le terrain m'a ouvert les yeux: suite au formidable effort du Tamil Nadu pour que ses indicateurs statistiques cessent d'être négatifs, on peut choisir une vision optimiste.

24 - EC. Le point de vue qui est celui de la classe moyenne occidentale est un point de vue biaisé, nous avons beaucoup de préjugés, comme certains enseignants qui sans s'en rendre compte sont presque insultants avec les enfants de milieux défavorisés. Quand on étudie les pays en voie de développement notre regard est plein de préjugés. Pour être un bon géographe, il faut se débarrasser de ses préjugés nationaux, sociaux et de genre... enfin essayer. Les sociétés traditionnelles sont fondées sur l'inégalité et l'oppression de tous les dominés. L'égalité reste une idée révolutionnaire aujourd'hui. Dans tous les pays du Sud, aujourd'hui, les ouvrières textiles vivent la même situation d'oppression que les ouvrières anglaises du XIX ${ }^{e}$ siècle. Ce qui me fait penser à une de mes interviewées, Maria, d'origine portugaise, arrivée en France en 1972, et qui a été femme de ménage toute sa vie. Pour nous, c'est un métier difficile et dévalorisé, mais elle ne le voyait pas comme ça : elle avait aimé passer l'aspirateur, parce qu'au Portugal elle n'avait pas l'électricité. Et son travail lui avait permis de passer son permis, d'acheter une voiture, de vivre à sa guise.

25 - EPG. J'ai particulièrement apprécié la manière dont vous rendez visible la parole des femmes immigrées dans votre dossier abc.immigration. Vous expliquez dans votre ouvrage qu'elles représentent aujourd'hui $49 \%$ des migrants, bénéficiant d'une offre de travail ciblée sur le care avec le vieillissement de la population des pays du Nord. On pense aux infirmières keralaises, étudiées par Marie Percot (2004), aux domestiques philippines, sri-lankaises et indonésiennes qui sont plus d'un million en Arabie 
Saoudite. Comment peut-on comprendre la citation que vous faites d'Alain Tarrius « Là où nous parlons exil, elles pensent projet »?

- EC. À la suite de mes interviews de femmes migrantes (abc.immigration) c'est ce que je pensais déjà, et la lecture de l'ouvrage collectif Le sexe de la mondialisation (2010), m'a apporté une confirmation : Liane Mozère a interviewé des Philippines émigrées à Paris et qui sont séparées de leurs enfants élevés par les grands-mères. Elle relève qu'elles acquièrent une certaine autonomie et se vivent comme des femmes qui construisent leur vie. Elles souffrent de la séparation, mais elles sont dans la réalisation d'un projet où elles vont changer la vie de leur famille. Il $\mathrm{y}$ a aussi un article dans Les Cahiers $d u$ Genre $\left(n^{\circ} 40,2006\right)$ sur des ouvrières d'Amérique Centrale parfaitement conscientes d'être exploitées, de travailler dans de mauvaises conditions, mais heureuses d'améliorer la vie de leur famille et leur statut. Là où nous voyons le négatif, elles voient le positif. Là où nous parlons exil, où nous insistons sur ce qu'il $\mathrm{y}$ a de douloureux, elles parlent du côté positif de leur projet. D'ailleurs, elles envoient une plus grande part de leur salaire que les hommes, avec une plus grande régularité et sur un temps plus long.

27 - EPG. Si l'on reprend l'exemple de l'Inde, en 1984, devant l'importance des capitaux provenant de la diaspora, le Premier ministre Rajiv Gandhi reconnait officiellement l'existence de la diaspora indienne. Dans un double mouvement d'internationalisation et de transnationalisation, comment les femmes de la diaspora peuvent-elles jouer un rôle de "community manager»? Quel est le vécu de l'émigration de ces femmes quand elles reviennent? Et quelles sont les conséquences de l'émigration pour celles qui restent?

28 - EC. Il est probable que lorsqu'une femme revient dans son pays d'origine, son statut a changé. Elle vit son émigration comme un levier de transformation. Elles sont allées affronter la solitude et l'inconnu. Elles partent pauvres et reviennent plus riches, elles partent jeunes et elles reviennent âgées, leur place dans la famille n'est plus la même puisqu'elles ont fait vivre plusieurs personnes en envoyant de l'argent, elles ont acquis de l'assurance. Elles ont vu autre chose, cela peut leur valoir un certain prestige.

29 Sans doute y a-t-il une grande différence entre les femmes qualifiées et celles qui ne le sont pas, entre celles qui ont migré dans des pays du Nord et celles qui ont migré dans des pays du Sud, entre celles qui ont bien réussi et celles qui ont été moins heureuses.

En tout cas, les pays de départ comptent sur elles, puisque les Philippines par exemple forment des infirmières pour l'émigration. La majorité des Philippins émigrés sont des femmes.

31 Pour celles qui restent, les situations sont extrêmement variées et dépendent de leur position vis-à-vis de la famille. Si au départ de leur mari, elles vivent au village dans leur belle-famille, elles ne doivent pas gagner beaucoup en autonomie. Mais si elles vivent en ville, seules avec leurs enfants, elles sont obligées de régler la vie courante toutes seules. La communauté, c'est protecteur mais c'est aussi oppressant. Je me souviens d'une femme interviewée qui disait: «je suis partie car je ne supportais plus le poids de la famille, je devais fuir cette honte ». Quelles sont vos observations sur ce point au Tamil Nadu?

32 - EPG. Sur mon terrain, il y a ce cas très particulier des femmes de culture tamoule et de nationalité française. Lors du traité de 1962, les habitants de Pondichéry devaient choisir s'ils voulaient être citoyen indien ou, en renonçant, choisir la nationalité 
française. La plupart de ces derniers se sont engagés dans l'armée française ou l'administration coloniale, puis sont revenus vivre leur retraite en famille à Pondichéry. Aujourd'hui, la nationalité française peut être vue comme un sésame pour entrer en France et en Europe. Certaines personnes constituent encore des dossiers pour justifier qu'ils n'ont pu se déplacer physiquement dans les délais impartis et renoncer à la nationalité indienne, et par conséquent obtenir la nationalité française pour eux et leurs descendants. Des jeunes filles de culture tamoule, ayant vécu toute leur vie à Pondichéry, se trouvent, elles, mariées à des Indiens à qui elles apportent en dot la nationalité française. Le choix de migrer est quasiment toujours masculin, c'est le plus souvent le mari qui cherche le logement et un emploi. La plupart des jeunes épouses partent en France avec leur mari et leur installation dépend beaucoup de leur maîtrise de la langue française. Parfois des situations singulières se dégagent. Des jeunes femmes arrivées avant leur mari, le temps que les papiers se fassent, se retrouvent à gérer seules, et pour la première fois, des entrées d'argent car les allocations sont versées à leur nom. D'autres sont accueillies par des membres de la belle-famille et elles restent souvent cantonnées à l'appartement familial. D'autres profitent de vacances à Pondichéry pour y rester avec leurs enfants et refusent de repartir en France. Il y a au consulat de Pondichéry une assistante sociale qui les aide pour que les enfants soient bien inscrits au lycée français gratuitement grâce aux dossiers de bourses, qu'ils bénéficient des allocations de rentrée et des centres de loisirs. Souvent ces femmes dépendent de l'argent envoyé, ou non, par le mari resté en France et un des projets du consulat m'a interpellé. Ces femmes sont dépendantes financièrement et très peu ont déjà travaillé à l'extérieur de leur domicile. Grâce à l'aide du consulat, elles reçoivent quelques notions de diététique. Elles peuvent ainsi préparer des repas adaptés à leur domicile pour qu'ils soient ensuite livrés chez des personnes âgées qui sont de plus en plus nombreuses à vivre sans leur famille. Pour ces femmes qui n'ont pour la plupart jamais travaillé à l'extérieur de leur domicile, c'est un bon début d'autonomisation.

- EC. Les études sur la condition féminine et les relations femmes-hommes, ou études de genre sont, à mes yeux, très importantes pour comprendre progrès et problèmes du monde actuel. À travers la condition féminine, on aborde l'anthropologie, l'histoire, l'économie, la culture, etc. C'est un bon angle d'attaque pour comprendre le monde actuel. Et ce que vous m'apprenez sur les femmes au Tamil Nadu, sur les efforts faits pour elles et par elles, fait partie des bonnes nouvelles, alors que comme vous l'avez dit, on ne connait l'Inde que par son actualité négative. C'est mon cas, même si je connais le travail des féministes indiennes. Il me semble que notre échange montre bien la complémentarité entre le travail sur les statistiques et le travail de terrain, le second corrigeant ou nuançant le premier.

\section{BIBLIOGRAPHY}

Boni T., 2008. Que vivent les femmes d'Afrique ? Paris, Éditions du Panama, 260 p. 
Borgeaud-Garcianda N., 2006. La vie sous-traitée des ouvrières des maquilas du Nicaragua. Cahiers du genre, $n^{\circ} 40,272 \mathrm{p}$.

Coquery-Vidrovitch C., 2013. Les Africaines. Paris, La découverte, 406 p.

Crémieu E., 2014. Géopolitique de la condition féminine. Paris, PUF, 224 p.

Crémieu E., http://abc.immigation.free.fr

Falquet J., Hirata H., Kergoat D., Labari B., Le Fleuvre N., Sow F., 2010. Le sexe de la mondialisation. Paris, Presses de Sciences Po, 280 p.

Guérin I., 2015. La micro-finance et ses dérives. Emanciper, discipliner ou exploiter ? Paris, Demopolis, $291 \mathrm{p}$.

Guérin I., Marius-Gnanou K., Servet J.M., Pairault T., 2005. Microfinance en Asie : entre traditions et innovations. Paris : Karthala, $230 \mathrm{p}$.

Kouvouama A., 2002. Modernité africaine. Figures du politique et du religieux. Brazaville, Edition Paari, $188 \mathrm{p}$.

Percot M., 2004. Les infirmières indiennes émigrés dans les pays du Golfe : de l'opportunité à la stratégie. REMI, vol. 21, $\mathrm{n}^{\circ} 1$, p. 29-54.

Ponceaud-Goreau E., 2015. Why go back to Pondicherry? French Tamil women in the return migration. In DESI, $\mathrm{n}^{\circ} 3$, "Indian values : diaspora and womanhood" edited by Anthony GoreauPonceaud and Paul Veyret, p. 51-68.

Racine J., 1994. Une vie paria : le rire des asservis, Inde du Sud. Paris, Plon, $636 \mathrm{p}$

Tawa-Lama Rewal S., 2004. Femmes et politique en Inde et au Népal, image et présence. Paris, Karthala, $337 \mathrm{p}$.

Van Woerkens M., 2010. Nous ne sommes pas des fleurs. Deux siècles de combats féministes en Inde. Paris, Albin Michel, 363 p.

\section{NOTES}

1. Reprenant la conception idéalisée de l'assemblée traditionnelle de village, c'est un système rural de représentation électif qui institue des conseils représentatifs au niveau local, ils sont les interlocuteurs des autorités pour mener à bien les politiques de développement.

\section{AUTHORS}

\section{ÉLIZABETH CRÉMIEU}

Elizabeth Crémieu, elizabethcremieu@orange.fr

\section{ÉMILIE PONCEAUD-GOREAU}

Émilie Ponceaud-Goreau, emilie.ponceaud@etu.u-bordeaux-montaigne.fr 